\title{
Covariance Analysis for Practical Identifiability of an Alveolar Re- cruitment Model
}

C. Schranz, K. Moeller, Institute for Technical Medicine, Furtwangen University, Villingen-Schwenningen, Germany, scc@hs-furtwangen.de

P. D. Docherty, Y. S. Chiew, J. G. Chase, Centre for Bioengineering, University of Canterbury, New Zealand

\section{Introduction}

Patient-specific mathematical models of respiratory mechanics can offer substantial insight into patient state and pulmonary dynamics that are not directly measurable. To assure bedside-applicability, the models must be computationally efficient and practically identifiable from the limited available data, while capturing dominant dynamics. This investigation measures the identifiability of a respiratory model for ARDS patients.

\section{Methods}

A recently developed pressure-dependent recruitment model, whose structural identifiability was proven, is fitted to low-flow test responses of 12 ARDS patients by a gradient-based parameter identification method. The reported parameter values are checked by covariance analysis. Each model parameter was declared constant at $10 \%$ of its reported minimum and the remaining parameters were re-identified. If parameter variance does not increase model fit-to-data error, it then may be concluded that the associated variable is non-identifiable. Non-identifiability might be caused by either limited effect of the model parameter on the model output or by another variable completely compensating the change.

\section{Results}

Identified parameter values were physiologically plausible and capable of reproducing the measured pressure responses with high accuracy. The covariance analysis of the results revealed premature parameter identification convergence and limited influence of particular model parameters during parameter identification. In particular, resistance and distension parameters were practically non-identifiable in some cases. These cases were recognisable by a lack of distension in the measured clinical data. Practical identifiably could be improved by either applying population values for particular parameters, ensuring sufficient information content is gathered from the clinical experiment, or reformulating the model.

\section{Conclusion}

The covariance analysis helped to validate reported results of parameter identification algorithms and was able to assess practical identifiability of the postulated model with given data. 